\title{
Determination of the Surface Properties of Alkali-Activated Slag by Inverse Gas Chromatography
}

\author{
Şafak Bilgiç, Serhat Çelikten, Mehmet Canbaz and Ceyda Bilgiç
}

\begin{abstract}
Inverse gas chromatography (IGC) has been used to measure the surface adsorption of some probes (n-alkanes on a series (n-C7 to $\mathrm{n}-\mathrm{C} 10)$ on Alkali-Activated Slag (AAS). The dispersive component of the surface energy $\left(\gamma_{S}^{d}\right)$, and the acid/base character of AAS surface were estimated by using the retention time of different non-polar probes at infinite dilution region. Dispersive component of the surface energy of AAS and the acid/base character were calculated between 60 and $90{ }^{\circ} \mathrm{C}$. The specific free energy of adsorption ( $\Delta G^{s p}$ ), the specific enthalpy of adsorption $\left(\Delta H^{s p}\right.$ ), and the specific entropy of adsorption $\left(\Delta S^{s p}\right.$ ) of polar probes on AAS were determined. $\Delta G^{s p}$ were correlated with the donor and modified acceptor numbers of the probes to quantify the acidic $\mathrm{K}_{\mathrm{A}}$ and the basic $\mathrm{K}_{\mathrm{D}}$ parameters of the AAS surface.
\end{abstract}

Keywords-Slag, Alkali Activation, Surface properties, Inverse gas chromatography (IGC).

\section{INTRODUCTION}

Durability is one of most important properties of cementitious materials because it is associated with the life service of civil engineering structures, and is modified by the interaction of the material with the environment. It is now accepted that new binders are needed to replace ordinary Portland cement (OPC) for enhanced environmental and durability performance. The urge to reduce emissions of carbon dioxide (1 tonne of OPC generates almost 1 tonne of $\mathrm{CO}_{2}$ ) and the fact that OPC structures, which have been built a few decades ago, are already facing disintegration problems points out the handicaps of OPC binders. In fact, the number of premature cases of OPC structures disintegration is overwhelming. Mehta [1] mentioned a case of pile foundations disintegrated just after 12 years and also the case of a tunnel in

Şafak Bilgiç, Department of Civil Engineering, Faculty of Engineering and Architecture, Eskişehir Osmangazi University, 26480 Eskişehir, Turkey

Serhat Çelikten, Dep. of Motor Vehicles and Transportation Tech., Vocational School of Transportation, Eskişehir Technical University, 26470, Eskişehir, Turkey

Mehmet Canbaz, Department of Civil Engineering, Faculty of Engineering and Architecture, Eskişehir Osmangazi University, 26480 Eskişehir, Turkey

Ceyda Bilgiç, Department of Chemical Engineering, Faculty of Engineering and Architecture, Eskişehir Osmangazi University, 26480 Eskişehir, Turkey
Dubai, which was concluded in 1975 and needed to be completely repaired in 1986. Gjorv [2] mentioned a study about Norway OPC bridges which indicates that $25 \%$ of those built after 1970 presented corrosion problems. Another author mentioned that $40 \%$ of the 600.000 bridges in the US were affected by corrosion problems, being the cost of the repairing operations estimated in 50 billion dollars [3].

Beyond the durability problems originated by imperfect concrete placement and curing operations, the real issue about OPC durability is related to the intrinsic properties of the material. Presents a higher permeability that allows water and other aggressive elements to enter concrete, leading to carbonation and corrosion problems. It also presents a higher amount of calcium hydroxide, which reacts with acids generating soluble compounds. So far, research works carried out in developing alkali activated binders show that this new binder is likely to have enormous potential to become an alternative to Portland cement. These binders are still at the early stage of development and, hence, need further research work in order to become technically and economically viable construction materials.

The essential requirements of a substance to be used in slag alkaline activation are: (a) acceleration of the solubility of slag; (b) promotion of the formation of stable hydrates; and (c) formation of a network structure of hydrates. There are few chemical compounds that fit these conditions and that can be used in the alkaline activation process of granulated blast furnace slag [4]. When compared with Portland cements and concretes, alkali-activated Slag (AAS) cements and concretes present several advantages, such as earlier and higher strengths, lower hydration heat, and stronger resistance to chemical attack. However, they also have some disadvantages, such as quick setting and high shrinkage rate with formation of microcracking [5]. The strength development and drying shrinkage of AAS are determined by the nature of the compounds forming during the activation and curing process [6]. Collins and Sanjayan [7] examined the effects of internal curing of AAS concrete by replacing normal weight coarse aggregate with fully saturated slag coarse aggregate and showed that the compressive strength was improved and the drying shrinkage was less. Puertas et al. [5] found that the nature of the matrix is the most important factor to the strength development. Krizan and Zivanovic [8] reported that the 
compressive strength of normal-cured water glass slag cements is higher than that of Portland cement mortars and drying shrinkage of alkali-slag cements is also higher than that of Portland cement. Bakharev et al. [9] studied the effect of elevated curing temperature on properties of AAS concrete and concluded that heat treatment greatly accelerates strength development, but at later ages the compressive strength was reduced compared to concrete cured at room temperature. In addition, heat curing considerably reduces shrinkage of AAS concrete. Fern'andez-Jim'enez et al. [10] studied the flexural and compressive strengths of AAS mortars and reported that liquid sodium silicate gave the highest strengths. The factors influencing the flexural and compressive strengths were the concentration of the activator, followed by curing temperature, and the fineness of the slag.

To understand the characteristics and composition of the solid surface, the surface characterization tools are required. Many techniques such as, X-ray photoelectron spectroscopy (XPS), zeta potential measurements, microcalorimetry, titration, contact angle and inverse gas chromatography (IGC) have been used to obtain detailed information on solid surfaces. IGC has frequently been applied for surface characterization of solids, in particular, for their thermodynamic behavior, surface energy and their Lewis acidic-basic character. Knowledge of the surface free energy at a given temperature and surface enthalpy of a solid has recently allowed significant progress in the fields of adsorption, wettability, catalysis, friction, and adhesion. The surface energy can be obtained by, liquid adsorption measurements, flow microcalorimetry or contact angle measurements, but these methods are not easily and widely applicable and are difficult to use for finely powder solids. IGC method is based on the study of physical adsorption of appropriate molecular probes by means of chromatographic (dynamic) experiments. In contrast to static methods, dynamic systems utilize a flowing gas system. The most common flow methods are IGC, gravimetric instruments, and permeability measurement systems. The principle of dynamic gravimetric systems is the measurement of the amount of solute adsorbed from a flowing gas stream using a microbalance. In comparison to static sorption equipment, the main benefits of the dynamic sorption technique are shorter measurement time and a wider range of experimental possibilities. Compared to other techniques (static measurements), IGC has attracted a lot of attention because of its simplicity and the rich information provided [11].

In the IGC the Alkali-Activated Slag to be characterized is used as the stationary phase, and a solute with well-known properties is injected as a probe. Several thermodynamic quantities, including surface energy, can thus be derived from the retention volume of probe passing through the column filled with Alkali-Activated Slag. This work was thus undertaken in order to investigate how IGC could be applied in estimating dispersive components of surface energies of Alkali-Activated Slag. Another purpose was to determine the variation in adsorption thermodynamic parameters of some hydrocarbons on Alkali-Activated Slag.

\section{II.MATERIALS AND METHODS}

\section{A. Materials}

The ground granulated blast furnace slag as a precursor for alkali activation was supplied from Kardemir iron and steel plant in Karabük, Turkey. The properties of the slag were given in the Table 1.

TABLE I

PROPERTIES OF SLAG

\begin{tabular}{|c|c|c|}
\hline \multirow{7}{*}{$\begin{array}{l}\frac{\widehat{d}}{0} \\
\tilde{0} \\
0 \\
0 \\
0\end{array}$} & $\mathrm{MgO}$ & 8.94 \\
\hline & $\mathrm{CaO}$ & 33.20 \\
\hline & $\mathrm{SiO}_{2}$ & 33.60 \\
\hline & $\mathrm{Al}_{2} \mathrm{O}_{3}$ & 9.88 \\
\hline & $\mathrm{Fe}_{2} \mathrm{O}_{3}$ & 1.05 \\
\hline & $\mathrm{SO}_{3}$ & 0.84 \\
\hline & LOI & 0.40 \\
\hline \multicolumn{2}{|c|}{ Spec. Gravity } & 2.85 \\
\hline \multicolumn{2}{|c|}{ Fineness, $\mathrm{cm}^{2} / \mathrm{g}$} & 3890 \\
\hline
\end{tabular}

The slag was activated with two activator named as sodium silicate $\left(\mathrm{Na}_{2} \mathrm{SiO}_{3}\right)$ and sodium hydroxide $(\mathrm{NaOH})$. The two activators $\left(\mathrm{Na}_{2} \mathrm{SiO}_{3}\right.$ and $\left.\mathrm{NaOH}\right)$ were supplied from local resources. The $\mathrm{Na}_{2} \mathrm{SiO}_{3}$ used in the mixtures was in liquid form and the $\mathrm{NaOH}$ was in solid form. The standard Rilem Cembureau sand was used in this study for manufacturing mortar mixtures of alkali activated slag. And also, the Eskişehir tap water was utilized in the mortar mixtures.

The mortar specimens were produced with a mortar mixture that consists of $450 \mathrm{~g}$ slag, $1350 \mathrm{~g}$ standard sand and $225 \mathrm{~g}$ water. The $\mathrm{Na}_{2} \mathrm{SiO}_{3}$ and $\mathrm{NaOH}$ were used in the mortar mixture as to the mixture consist $5 \% \mathrm{Na}$ of slag content by weight. In addition, the Ms modulus (the total $\mathrm{Na}_{2} \mathrm{O} / \mathrm{SiO}_{2}$ ratio of activators) of the activators was adjusted as 0.5 in the mixtures. The $225 \mathrm{~g}$ water content was composed of the tap water and the water in the $\mathrm{Na}_{2} \mathrm{SiO}_{3}$. The specimens were cured at $80^{\circ} \mathrm{C}$ during 12 hours in an oven.

For the IGC analysis, the polar probes used were n-heptane, n-octane, n-nonane and n-decane. The polar probes used were tetrahydrofuran (THF), acetone (Acet.), diethyleter (DEE), trichloromethane (TCM), and ethyl acetate (Et.Ac.). All of these chemicals were analytical reagent grade and used without further purification. Properties were taken from literature of the probes [12]. These probes are commonly used for solid surface characterizations by IGC method. The chromatographic experiments were performed with Agilent 7890 gas chromatography equipped with a flame ionization detector (FID). A vacuum pump was used for packing the solids into the columns. High purity nitrogen was used as the carrier gas with a flow rate of about $40 \mathrm{~mL} / \mathrm{min}$. The flow rate 
of carrier gas was corrected for pressure drop and temperature change in the column using James-Martin gas compressibility factor. A stainless steel column (2.00 m long, $5.35 \mathrm{~mm}$ I.D.) previously washed with methanol and acetone was packed with AAS powders. The two ends of the column were plugged with silane-treated glass wool. Measurements were carried out in the temperature range of $60-90{ }^{\circ} \mathrm{C}$. The column was stabilized overnight in stream of nitrogen at $150{ }^{\circ} \mathrm{C}$. The dead volumes of the columns were determined by injecting methane. At least three replicate determinations were used in averaging the net retention volume $\left(\mathrm{V}_{\mathrm{N}}\right)$.

\section{B. Calculations}

IGC is used to study the surface free energy and acid-base properties of solids; the study is generally performed in the area of infinite dilution where the interactions between the adsorbed probe molecules are negligible. IGC provides an excellent method to measure the surface energy of rough and porous powders [13]. The surface energy is the result of the unbalanced molecular forces at the surface of the solid. It can be considered to be formed by two different contributions: dispersive and specific [14]. The retention time of a series of homologous n-alkanes is used to determine the dispersive component of the surface free energy $\left(\gamma_{s}^{d}\right)$ of AAS. In IGC literature, $\gamma_{S}^{d}$ is commonly determined from the following equation, which was introduced by Lavielle and Schultz [12]:

$$
R T \operatorname{Ln} V_{N}=2 N \cdot\left(\gamma_{S}^{d}\right)^{1 / 2} \cdot a \cdot\left(\gamma_{L}^{d}\right)^{1 / 2}+C
$$

Here, $\mathrm{R}$, is the gas constant, $\mathrm{T}$, is the absolute column temperature $(\mathrm{K})$, a, is the molecular surface area coated with a kind of adsorbed alkane, $\mathrm{N}$, is the Avogadro's number, $\gamma_{L}^{\mathrm{d}}$, is the dispersive component of the surface free energy of the probe, $\mathrm{C}$ is a constant and $\mathrm{V}_{\mathrm{N}}$ is the net retention volume of the $\mathrm{n}$-alkane probe. The net retention volume $\left(\mathrm{V}_{\mathrm{N}}\right)$ is calculated using the equation below [15]:

$$
\mathrm{V}_{N}=\mathrm{F}_{\mathrm{o}} \frac{\mathrm{T}}{\mathrm{T}_{\mathrm{o}}} \frac{3}{2}\left[\frac{\left(\mathrm{P}_{i} / \mathrm{P}_{\mathrm{o}}\right)^{2}-1}{\left(\mathrm{P}_{i} / \mathrm{P}_{o}\right)^{3}-1}\right]\left(\mathrm{t}_{\mathrm{A}}-\mathrm{t}_{0}\right)
$$

Here, $t_{\mathrm{A}}$ is the retention time of the probe, while to is the retention time of the probe which has no interaction with the solid in the column (marker). $\mathrm{P}_{\mathrm{i}}$ and $\mathrm{P}_{\mathrm{o}}$ are, respectively, the inlet and outlet pressures of the carrier gas, while the $T / T_{o}$ ratio is used in order to get the value of the flow rate at the column temperature $(\mathrm{T})$ from the measurement of the flow rate at ambient temperature $\left(\mathrm{T}_{\mathrm{o}}\right)$. The flow rate of the carrier gas measured at the column outlet and at ambient temperature is expressed as $\mathrm{F}_{\mathrm{o}}$.

The values of $K_{A}$ and $K_{D}$ were calculated using Equation (3) [16]:

$$
-\frac{\Delta H^{s p}}{A N^{*}}=K_{A} \frac{D N}{A N^{*}}+K_{D}
$$

Here, $\Delta \mathrm{H}^{\mathrm{sp}}$ is the specific component of the adsorption enthalpy and the $K_{A}$ and $K_{D}$ parameters define acid and base constants of the analyzed surface, respectively, $\mathrm{AN}^{*}$ shows the acceptor number of the adsorbed probe while DN shows the donor number. The $\mathrm{K}_{\mathrm{A}}$ and $\mathrm{K}_{\mathrm{D}}$ values can be calculated with the aid of probes which have acidic, basic, and amphoteric properties. According to the above equation, representing the $\left(\Delta \mathrm{H}^{\mathrm{sp}} / \mathrm{AN}^{*}\right)$ versus $\left(\mathrm{DN} / \mathrm{AN}^{*}\right)$, one gets $\mathrm{K}_{\mathrm{A}}$ as the slope and $\mathrm{K}_{\mathrm{D}}$ as the intercept. Finally, $\mathrm{K}_{\mathrm{D}} / \mathrm{K}_{\mathrm{A}}$ is known as $\mathrm{S}_{\mathrm{C}}$. According to the values obtained from these parameters, it is accepted that $\mathrm{S}_{\mathrm{C}} \leq 0.9$ means the surface is acidic, $\mathrm{S}_{\mathrm{C}} \geq 1.1$ means basic, and if it is between 0.9 and 1.1 , the surface is amphoteric [17].

\section{RESULTS AND DISCUSSION}

The chromatographic peaks were symmetric and had maxima that were independent of the amount injected. It is assumed that the adsorption occurs in the Henry's law region (at zero surface coverage) where lateral interactions between the adsorbates at the surface can be neglected. The net retention volumes, $\mathrm{V}_{\mathrm{N}}$, were obtained from the maxima of the chromatographic peaks and the dead time volume Equation (2).

The dispersive component of the surface free energy, $\gamma_{S}^{d}$, was determined by injection of a homologous series of $n$ alkanes having between 7 and 10 carbon atoms. One of the most commonly measured parameters for the description of the energy situation on the surface of a solid is the surface energy. The surface energy can affect, e.g. catalytic activity or the strength of particle-particle interaction. The dispersive components of AAS at experimental temperatures were calculated from Equation (1), (Figure 1). The variation of $\gamma_{S}^{d}$ calculated as a function of temperature. It was observed that $\gamma_{S}^{d} \quad$ values decrease with temperature. The obtained $\gamma_{S}^{d}$ values for AAS were ranged from $44.44 \mathrm{~mJ} . \mathrm{m}^{-2}$ at $90^{\circ} \mathrm{C}$, $53.162 \mathrm{~mJ} . \mathrm{m}^{-2}$ at $80^{\circ} \mathrm{C}, 57.15 \mathrm{~mJ} . \mathrm{m}^{-2}$ at $70^{\circ} \mathrm{C}$, and 62.45 $\mathrm{mJ} . \mathrm{m}^{-2}$ at $60^{\circ} \mathrm{C}$.

A comprehensive insight into the Lewis acid-base surface interactions provides a better understanding of the influence of the catalytic and sorption capabilities of AAS, as well as their ability to change via chemical modifications, which is of great importance for their applications. The application of the Schultz's method to our experimental results (Figure 1 and 2) gave us the value of $\Delta G^{s p}$. Polar probes were used for the investigation of acid-base nature of the surfaces. The free energy of the acid-base interactions of polar probes with the samples, $\Delta G^{s p}$, was calculated. Table 1 shows the free energy of the specific interactions between the sample and polar probes at different temperatures. It was observed that; the $\Delta G^{s p}$ value decreases the temperature increases. The 
average $-\Delta G^{s p}$ values of polar probes for kaolinite in the following order: $\mathrm{TCM}<\mathrm{THF}<\mathrm{Et}$. Ac $<\mathrm{DEE}<$ Acetone. The lowest $\Delta G^{s p}$ was observed with the acidic TCM solvent. It can be observed that for AAS, probes with large DN number (such as THF and acetone) show higher $\Delta G^{s p}$ values. Very low values can be observed for TCM, implying that although the adsorption mechanism of this molecule is considered as a combined mechanism, the porosity is much more important than the chemical surface groups because the specific contribution.

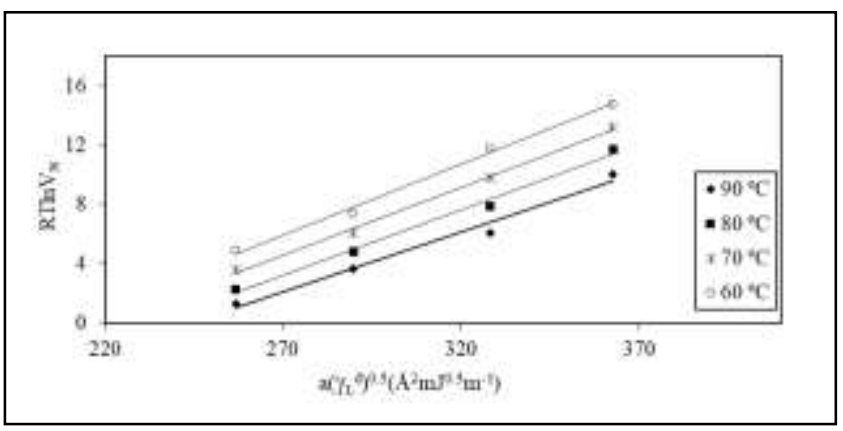

Fig. 1 The $R T \ln \left(V_{N}\right)-a \cdot\left(\gamma_{L}^{d}\right)^{1 / 2}$ graphs for AAS.

TABLE II

THE VARIATION OF $\Delta \mathrm{G}^{\mathrm{SP}}$ WITH TEMPERATURE, AND $\Delta \mathrm{H}^{\mathrm{SP}}$ AND $\Delta \mathrm{S}^{\mathrm{SP}}$ VALUES OF POLAR PROBES ON AAS

\begin{tabular}{|l|c|c|c|c|c|c|}
\hline \multicolumn{5}{|c|}{$\Delta \mathrm{G}^{\mathrm{SP}}(\mathrm{kJ} / \mathrm{mol})$} & $\begin{array}{l}-\Delta \mathrm{H}^{\mathrm{sp}} \\
(\mathrm{kJ} / \mathrm{mol})\end{array}$ & $\begin{array}{l}-\Delta \mathrm{S}^{\mathrm{sp}} 10^{3} \\
(\mathrm{~kJ} / \mathrm{mol} . \mathrm{K})\end{array}$ \\
\cline { 1 - 5 } $\mathrm{T}\left({ }^{\circ} \mathrm{C}\right)$ & $60^{\circ} \mathrm{C}$ & $70^{\circ} \mathrm{C}$ & $80^{\circ} \mathrm{C}$ & $90^{\circ} \mathrm{C}$ & & \\
\hline TCM & 1.59 & 2.30 & 2.25 & 2.19 & 4.31 & -18.39 \\
\hline DEE & 5.27 & 5.33 & 5.73 & 5.21 & -5.64 & 9.22 \\
\hline Acet. & 7.85 & 7.95 & 8.13 & 7.64 & -9.16 & 3.63 \\
\hline THF & 4.18 & 4.20 & 4.22 & 4.06 & -5.31 & 3.30 \\
\hline Et. Ac. & 4.99 & 5.07 & 5.28 & 4.88 & -5.37 & 8.80 \\
\hline
\end{tabular}

The values of the specific enthalpy of adsorption, $\Delta H^{s p}$ and the specific entropy of adsorption, $\Delta S^{s p}$ computed by varying $\Delta G^{s p} / T$ against $1 / T$ are presented in Table 1. By ploting $-\Delta H^{s p} / A N^{*}$ versus $D N / A N^{*}$, according to Equation $3 K_{A}$ and $K_{D}$ can be obtained from the slope and the intercept, respectively (Figure 2). The values of $K_{A}$ and $K_{D}$ for AAS are found to be 0.056 and 0.295 . According to the values obtained from $K_{D} / K_{A}=5.26$ is reflected the surface of AAS is basic.

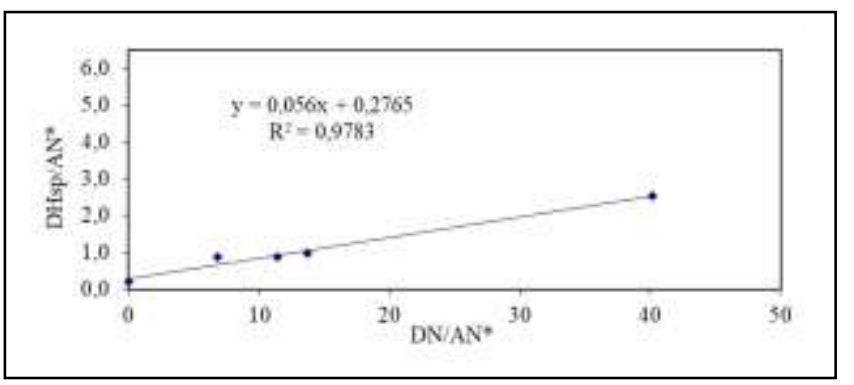

Fig. 2 Plots of $-\Delta H^{s p} / A N^{*}$ versus $D N / A N^{*}$ for the adsorption of organic probes on AAS.

\section{CONCLUSION}

IGC as an independent field of study has certainly proven beneficial to many industries. Despite a long history and several hundred publications in the last 50 years, it is still evolving and considered a modern technique that is quite attractive to chemical engineers, analytical chemists and material scientists in various fields. Results of a large quantity of research experiments on a wide range of materials have evidenced that IGC is successfully applied to characterizing different surface and bulk properties of solids in different shapes and morphologies. One of the greatest advantages of this method is that no special sample preparation is required. In fact, IGC requires the minimum sample preparation when compared to other surface energy analyzing techniques. Therefore, various forms of solids and even semi-solids can be characterized quickly and efficiently.

In this study, showed that IGC is a powerful analytical technique very useful for studying the surface properties of AAS and for monitoring adsorption processes. Surface information on adsorption was obtained from the temperature variation of the partition coefficients for probes at zero coverage. The dispersive component of free energy of adsorption $\gamma_{S}^{d}$ probably was related to structural heterogeneities on the lateral surfaces, as well as to the channels and pores present at $60-90{ }^{\circ} \mathrm{C}$. The experimental results indicate that $\gamma_{S}^{d}$ values of studied materials gradually decreased with increasing column temperature which is consistent with the fundamental concept of Gibbs free energy. The values of $\mathrm{K}_{\mathrm{A}}$ and $\mathrm{K}_{\mathrm{D}}$ indicated that AAS surface is basic. Overall, the results showed that IGC is an effective method to characterize the AAS surface properties.

\section{REFERENCES}

[1] P.K. Mehta, "Concrete in marine environment," New York, USA: Elsevier Science Publishers; 1991.

[2] O.E. Gjorv, "Steel corrosion in concrete structures exposed to Norwegian marine environment," ACI. Concr. Int., pp. 35-39, 1994.

[3] Ferreira Rui Miguel, "Tests for evaluation of concrete durability," Master thesis, University of Minho; 2000 [only in Portuguese].

[4] K. Darko and Z. Branislav, "Effects of dosage and modulus of water glass on early hydration of alkali-slag cements," Cement and Concrete Research, vol. 32, pp. 1181-1188, 2001.

[5] F. Puertas, T. Amat, A. Fern'andez-Jim'enez, and T. V'azquez, "Mechanical and durable behaviour of alkaline cement mortars reinforced with polypropylene fibres," Cement and Concrete Research, vol. 33, no. 12, pp. 2031-2036, 2003.

https://doi.org/10.1016/S0008-8846(03)00222-9

[6] Z. Vladimir, "Effects of type and dosage of alkaline activator and temperature on the properties of alkali-activated slagmixtures," Construction and Building Materials, vol. 21, no. 7, pp. 1463-1469, 2007.

https://doi.org/10.1016/j.conbuildmat.2006.07.002

[7] F. Collins and J. G. Sanjayan, "Strength and shrinkage properties of alkali-activated slag concrete containing porous coarse aggregate," Cement and Concrete Research, vol. 29, no. 4, pp. 607-610, 1999. https://doi.org/10.1016/S0008-8846(98)00203-8

[8] D. Krizan and B. Zivanovic, "Effects of dosage and modulus of water glass on early hydration of alkali-slag cements," Cement and Concrete Research, vol. 32, no. 8, pp. 1181-1188, 2002. https://doi.org/10.1016/S0008-8846(01)00717-7 
[9] T. Bakharev, J. G. Sanjayan, and Y.-B. Cheng, "Effect of elevated temperature curing on properties of alkali-activated slag concrete," Cement and Concrete Research, vol. 29, pp. 1619-1625, 1999. https://doi.org/10.1016/S0008-8846(99)00143-X

[10] A. Fern’andez-Jim’enez, J. G. Palomo, and F. Puertas, "Alkaliactivated slag mortars: mechanical strength behaviour," Cement and Concrete Research, vol. 29, no. 8, pp. 1313-1321, 1999.

[11] S. Mohammadi-Jam, K.E. Waters, "Inverse gas chromatography applications: a review, "Advanced Colloid Interface, vol. 212, pp. 2144, 2014. https://doi.org/10.1016/j.cis.2014.07.002

[12] N. Cordeiro, C. Gouveia, M.J. John, "Investigation of Surface Properties of Physico-Chemically Modified Natural Fibres Using Inverse Gas Chromatography," Industrial Crops and Products, vol. 33, pp. 108-115, 2011.

[13] B.B. Hole, D.S. Keller, W.M. Burry, and J.A. Schwarz, "Surface energetics of bone mineral and synthetic hydroxyapatite using inverse gas chromatography, "J. Chromatogr. B. 879, pp.1847-1850, 2011. https://doi.org/10.1016/j.jchromb.2011.04.035

[14] M. Perez-Mendoza, M.C. Almazan-Almazan, L. Mendez-Linan, M. Domingo-García, F.J. López-Garzón, "Evaluation of the dispersive component of the surface energy of active carbons as determined by inverse gas chromatography at zero surface coverage," J. Chromatogr. A., vol. 1214, pp. 121-127, 2008. https://doi.org/10.1016/j.chroma.2008.10.070

[15] J.F. Gamble, R.N. Dave, S. Kiang, M.M. Leane, M. Tobyn, S.S.Y. Wang, "Investigating the applicability of inverse gas chromatography to binary powdered systems: an application of surface heterogeneity profiles to understanding preferential probe-surface interactions," International Journal of Pharmaceutics. vol. 445, pp. 39- 46, 2013. https://doi.org/10.1016/j.ijpharm.2013.01.061

[16] S. Lazarevic, Z. Radovanovic, Dj Veljovic, A. Onjia, Dj Janackovic, R. Petrovic, "Characterization of sepiolite by inverse gas chromatography at infinite and finite surface coverage," Applied Clay Science, vol. 43, pp. 41-48, 2009. https://doi.org/10.1016/j.clay.2008.07.013

[17] D. Cava, R. Gavara, J.M. Lagaron, A. Voelkel, "Surface characterization of poly(lactic acid) and polycaprolactone by inverse gas chromatography, " J. Chromatogr. A., vol. 1148, pp. 86-91, 2007. https://doi.org/10.1016/j.chroma.2007.02.110 